\title{
Influence of the shape and size of a quantum wire on the trion binding energy
}

\author{
Y. Sidor, B. Partoens, ${ }^{*}$ and F. M. Peeters ${ }^{\dagger}$ \\ Departement Fysica, Universiteit Antwerpen, Groenenborgerlaan 171, B-2020 Antwerpen, Belgium \\ (Received 19 December 2007; revised manuscript received 11 April 2008; published 12 May 2008)
}

\begin{abstract}
The binding energy for charged excitons $\left(X^{-}\right.$and $\left.X^{+}\right)$is calculated within the single-band effective mass approximation including effects due to strain for rectangular, triangular, and V-shaped quantum wires. Both $X^{-}$ and $X^{+}$are found to be bound in rectangular InAs/InP quantum wires and V-shaped $\mathrm{GaAs} / \mathrm{Al}_{0.32} \mathrm{Ga}_{0.68} \mathrm{As}$ quantum wires. We found an appreciable dependence of the trion binding energy on the size and shape of the quantum wire. We compare with available experimental data.
\end{abstract}

DOI: 10.1103/PhysRevB.77.205413

PACS number(s): 73.21.Hb

\section{INTRODUCTION}

Negative $\left(X^{-}\right)$and positive $\left(X^{+}\right)$trions result from the binding of an exciton $(X)$ with an extra electron or hole, respectively. The existence of two kinds of trions was first suggested by Lampert. ${ }^{1}$ Due to the relatively small binding energy of trions in bulk semiconductors, they can only be observed at very low temperatures $(T<10 \mathrm{~K})$. Recent progress in semiconductor growth techniques has made it possible to fabricate low-dimensional nanostructures. The reduced dimensionality considerably increases the binding energy of trions, and thus facilitates the formation of $X^{-}$and $X^{+}$ in semiconductor quantum wells (QWs), quantum wires (QWRs), and quantum dots. ${ }^{2-16}$ It was shown that well width fluctuations in QWs will trap the trion and lead to a large increase of its binding energy. ${ }^{17-19}$

The purpose of this work is to investigate the stability of charged excitons (trions) in a QWR. Our work is motivated by the recent observation of negatively and positively charged excitons in V-shaped $\mathrm{GaAs} / \mathrm{Al}_{032} \mathrm{Ga}_{06}{ }_{68} \mathrm{As}$ QWRs. ${ }^{20}$ We will also study the formation of trions in self-assembled rectangular InAs/InP QWRs which were experimentally investigated in Refs. 21 and 22. In our model, we include the true geometrical shape of the $\mathrm{V}$-shaped and rectangular wires, the influence of strain on the particle confinement potentials in the self-assembled wires, as well as the mass mismatch between the wire and the barrier materials. We assume that the single particle energies due to the radial confinement are much larger than the Coulomb energies between the constituents of the trion. This allows us to use the adiabatic approximation which reduces the problem to the solution of a two-dimensional (2D) effective Schrödinger equation. The 2D calculations are based on a finite element method within the single-band effective mass approximation.

In previous work on trions confined in a QWR, a parabolic confinement potential was assumed. ${ }^{23}$ Such an assumption considerably simplifies the calculations but it is not able to model effects that result from the shape of the QWR. Recently, trions were also studied ${ }^{24}$ in circular freestanding wires. Effects due to the dielectric mismatch that renormalize the Coulomb interparticle interaction are very important in this system. The latter can be neglected in the present study of embedded wires where the well and barrier materials have almost the same dielectric constant.

The paper is organized as follows. In Sec. II, we describe our theoretical model and we give details about the approxi- mations that are used to solve it. In Sec. III, we present our numerical results. Our conclusions are given in Sec. IV.

\section{THEORETICAL MODEL}

The InAs/InP self-assembled QWR is modeled as a 2D rectangular quantum box with height $h$ and width $w$, as schematically shown in Fig. 1(a). We identify the crystallographic axes [110], [001], and [1 $\overline{1} 0]$ with the $x, y$, and $z$ axes of the wire. ${ }^{25-27}$ The height $h$ will be expressed in monolayers (MLs), and $1 \mathrm{ML}=3.03 \AA .{ }^{26,27}$ For the simulations of the V-shaped GaAs/ $\mathrm{Al}_{0.32} \mathrm{Ga}_{0.68} \mathrm{As}$ QWR, we use a profile with heights $h$ and $H$, and the base length $w$ [indicated by the white line in Fig. 1(b)], as suggested by the transmission electron microscopy (TEM) image of the wire in Ref. 28. We base our geometry for V-shaped QWRs on this TEM image, as similar QWRs were considered in Ref. 20 in which the binding energy of charged excitons was experimentally obtained. We will compare our numerical results later with the two QWRs considered in Ref. 20: the thick one with $h$ $=70 \AA$, and the thin one with $h=30 \AA$. The width of both QWRs was not given in Ref. 20, but based on the profile of the TEM image [indicated by the white line in Fig. 1(b)] of Ref. 28, we choose the widths of $w=510 \AA$ for the thick QWR and $w=210 \AA$ for the thin QWR. In order to investigate the effect of the shape on the trion binding energies, we also consider triangular QWRs with height $h$ and width $w$.

We assume that the conduction and highest valence bands are decoupled, which is a reasonable approximation for the

(a)
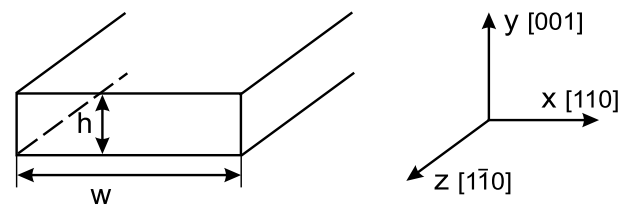

(b)
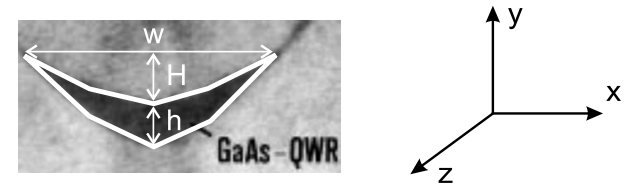

FIG. 1. Schematic view of (a) our model of rectangular selfassembled InAs/InP QWR with height $h$ and width $w$. (b) Geometry of V-shaped QWR with thicknesses $h$ and $H$ at its center, and the base length $w$, as suggested by TEM micrography in Ref. 28 . 
two considered types of wires because of the large direct band gap of the InAs and GaAs materials. The full Hamiltonian of a negative trion in a QWR, within the single-band effective mass approximation, is given by

$H_{X^{-}}=H_{e 1}+H_{e 2}+H_{h}-\frac{e^{2}}{\varepsilon\left|\mathbf{r}_{e 1}-\mathbf{r}_{h}\right|}-\frac{e^{2}}{\varepsilon\left|\mathbf{r}_{e 2}-\mathbf{r}_{h}\right|}+\frac{e^{2}}{\varepsilon\left|\mathbf{r}_{e 1}-\mathbf{r}_{e 2}\right|}$,

where $e 1$ and $e 2$ indicate the two electrons and $h$ the hole. $H_{e 1(2)}$ is the Hamiltonian of the confined first (second) electron, $H_{h}$ is the hole Hamiltonian, $e$ is the free-electron charge, and $\varepsilon$ is the dielectric constant taken as the average value of the one of the wire and the barrier. The last three terms in the full Hamiltonian are the electron-hole and electron-electron Coulomb interactions. Note that the Hamiltonian (1) is nine dimensional (9D). We are allowed to separate the $z$ motion from the lateral motion in the $x y$ plane because of the strong confinement in the $x y$ plane while there is no confinement for the particles along the wire direction $(z$ direction). In other words, we assume that the Coulomb interaction does not affect the $x y$ motion of the particles, so that we can separate the electrons and hole wave functions. In this case, the negative trion wave function can be written as

$$
\begin{aligned}
\Psi_{X^{-}}\left(\mathbf{r}_{e 1}, \mathbf{r}_{e 2}, \mathbf{r}_{h}\right)= & \Psi_{e 1}\left(x_{e 1}, y_{e 1}\right) \\
& \times \Psi_{e 2}\left(x_{e 2}, y_{e 2}\right) \Psi_{h}\left(x_{h}, y_{h}\right) \Phi\left(z_{e 1}, z_{e 2}, z_{h}\right),
\end{aligned}
$$

where $\Psi_{e 1}\left(x_{e 1}, y_{e 1}\right), \Psi_{e 2}\left(x_{e 2}, y_{e 2}\right)$, and $\Psi_{h}\left(x_{h}, y_{h}\right)$ are the electron and hole wave functions in the lateral directions, respectively, and $\Phi\left(z_{e 1}, z_{e 2}, z_{h}\right)$ is the wave function of the negative trion along the wire direction. Next, we averaged the 9D Schrödinger equation over the single particle wave functions $\Psi_{e 1}, \Psi_{e 2}$, and $\Psi_{h}$ in the confinement directions $x$ and $y$ and we are left with a differential equation describing the $z$ motion of the three particles. Then, we introduce the relative coordinates between the electrons and hole $z_{1(2)}=z_{h}$ $-z_{e 1(2)}$ and the center-of-mass (c.m.) coordinate $Z=\left(m_{e 1}^{*} z_{e 1}\right.$ $\left.+m_{e 2}^{*} z_{e 2}+m_{h}^{*} z_{h}\right) /\left(2 m_{e 1}^{*}+m_{h}^{*}\right)$. The center-of-mass momentum $K_{\text {c.m. }}$ is an integral of motion, which allows us to decouple the slow motion of the center of mass from the fast relative motion

$$
\Phi\left(z_{e 1}, z_{e 2}, z_{h}\right)=\phi_{X^{-}}\left(z_{1}, z_{2}\right) \exp \left(i K_{\text {c.m. }} Z\right) .
$$

Finally, we obtain the effective 2D Schrödinger equation for the relative motion of the negative trion

$$
\begin{aligned}
& {\left[-\frac{\hbar^{2}}{2 \mu_{z}}\left(\nabla_{z_{1}}^{2}+\nabla_{z_{2}}^{2}\right)-\frac{1}{m_{h}^{*}} \nabla_{z_{1}} \nabla_{z_{2}}+U_{e f f}^{(e 1-e 2)}\left(z_{1}-z_{2}\right)\right.} \\
& \left.\quad-U_{e f f}^{(e 1-h)}\left(z_{1}\right)-U_{e f f}^{(e 2-h)}\left(z_{2}\right)\right] \phi_{X^{-}}\left(z_{1}, z_{2}\right)=E_{X^{-}} \phi_{X^{-}}\left(z_{1}, z_{2}\right),
\end{aligned}
$$

where $\mu_{z_{1}}=\mu_{z_{2}}=\mu_{z}$ is the reduced mass of the electron and hole, which is equal to the reduced mass of a neutral exciton $\mu_{z^{\prime}} \cdot E_{X^{-}}$and $\phi_{X^{-}}\left(z_{1}, z_{2}\right)$ are the total energy and the relative
TABLE I. Input material parameters for InAs/InP and GaAs/ $\mathrm{Al}_{0.32} \mathrm{Ga}_{0.68} \mathrm{As}$ QWRs used in the calculations: lattice constant $a_{0}$, band gap $E_{g}$, electron mass $m_{e}^{*}$ (Refs. 29-31), Luttinger parameters $\gamma_{1}$ and $\gamma_{2}$, nonparabolicity parameters $\alpha$ (Refs. 32-34), the hydrostatic deformation potential for the conduction band $a_{c}$, the deformation potentials of the valence band $a_{v}, b$, and $d$, strain coefficients $C_{11}$ and $C_{12}$, and dielectric constant $\varepsilon$. For $\mathrm{Al}_{x} \mathrm{Ga}_{1-x} \mathrm{As}$ material parameters, the first-order interpolation formula is used: parameter $\left(\mathrm{Al}_{x} \mathrm{Ga}_{1-x} \mathrm{As}\right)=\operatorname{parameter}(\mathrm{GaAs}) \pm x($ parameter $(\mathrm{GaAs})$ parameter(AlAs)).

\begin{tabular}{lcccc}
\hline \hline Parameter & InAs & InP & GaAs & $\mathrm{Al}_{0.32} \mathrm{Ga}_{0.68} \mathrm{As}$ \\
\hline$a_{0}(\AA)$ & 6.058 & 5.869 & & \\
$E_{g}(\mathrm{eV})$ & 0.417 & 1.424 & 1.519 & 1.918 \\
$m_{e}^{*}\left(\mathrm{~m}_{0}\right)$ & 0.023 & 0.077 & 0.067 & 0.094 \\
$\gamma_{1}$ & 20 & 5.08 & 6.98 & 5.95 \\
$\gamma_{2}$ & 8.5 & 1.6 & 2.06 & 1.66 \\
$\alpha\left(\mathrm{eV}^{-1}\right)$ & 1.4 & & 0.64 & \\
$a_{c}(\mathrm{eV})$ & -5.08 & & & \\
$a_{v}(\mathrm{eV})$ & 1 & & & \\
$b(\mathrm{eV})$ & -1.8 & & & \\
$d(\mathrm{eV})$ & -3.6 & & & \\
$C_{11}(\mathrm{GPa})$ & 83.29 & & & \\
$C_{12}(\mathrm{GPa})$ & 45.26 & & & \\
$\varepsilon$ & 15.15 & 12.5 & 12.9 & \\
\hline \hline
\end{tabular}

wave function of the negative trion, respectively. The procedure of calculating the effective potentials $U_{\text {eff }}\left(z_{1}-z_{2}\right)$, $U_{\text {eff }}\left(z_{1}\right)$ and $U_{\text {eff }}\left(z_{2}\right)$ is identical to the one for the single exciton which we discussed previously in Ref. 27. Note that the problem is now reduced to solve a $2 \mathrm{D}$ differential equation. The latter was numerically realized by using the finite element method.

Similarly, we obtain the corresponding equation for the relative motion of the $X^{+}$

$$
\begin{aligned}
& {\left[-\frac{\hbar^{2}}{2 \mu_{z}}\left(\nabla_{z_{1}}^{2}+\nabla_{z_{2}}^{2}\right)-\frac{1}{m_{e}^{*}} \nabla_{z_{1}} \nabla_{z_{2}}+U_{e f f}^{(h 1-h 2)}\left(z_{1}-z_{2}\right)\right.} \\
& \left.-U_{e f f}^{(e-h 1)}\left(z_{1}\right)-U_{e f f}^{(e-h 2)}\left(z_{2}\right)\right] \phi_{X^{+}}\left(z_{1}, z_{2}\right)=E_{X^{+}} \phi_{X^{+}}\left(z_{1}, z_{2}\right),
\end{aligned}
$$

where $E_{X^{+}}$is the total energy of the positive trion. Note that in this case the definition for the relative positions of the first and the second hole with respect to the electron position, namely, $z_{1}=z_{h 1}-z_{e}$ and $z_{2}=z_{h 2}-z_{e}$, and for the center-ofmass coordinates $Z=\left(m_{e}^{*} z_{e}+m_{h 1}^{*} z_{h 1}+m_{h 2}^{*} z_{h 2}\right) /\left(m_{e}^{*}+2 m_{h 1}^{*}\right)$ are different from those for the $X^{-}$.

The input parameters used in our calculations are given in Table I. The values of the parameters have been taken from Ref. 35, or indicated otherwise. We include strain effects and the conduction band nonparabolicity following our earlier theoretical approaches of Refs. 27 and 36. The mass of the heavy hole is taken the same in each direction, i.e., $\mu_{h}^{*}$ 


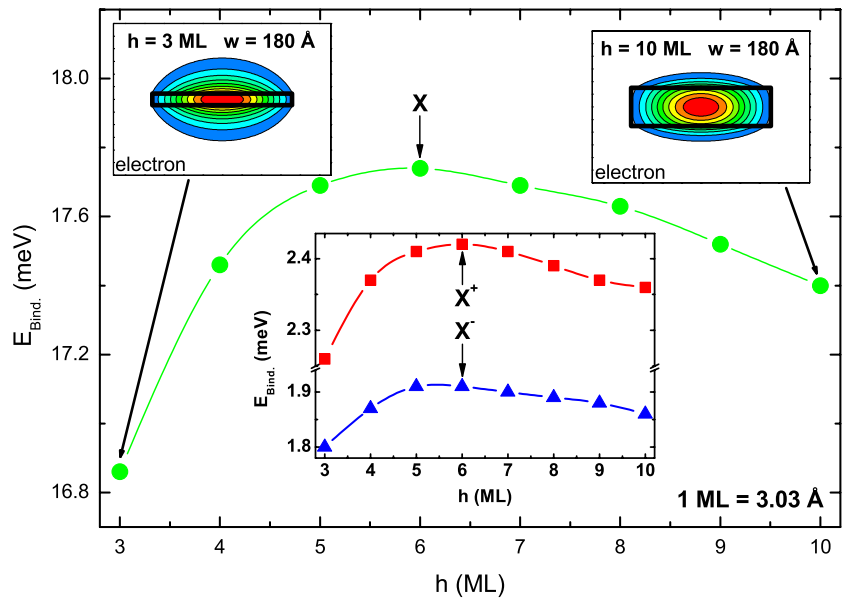

FIG. 2. (Color online) The exciton binding energy as a function of the height $h$ of the InAs/InP QWR with $w=180 \AA$ [see the curves with the circles (green)]. The insets show the binding energy of the positive trion $X^{+}$[see the curves with the squares (red)] and the negative trion $X^{-}$[see the curves with the triangles (blue)] as a function of the height $h$ of the InAs/InP QWR, and the contour plots of the electron wave functions when $h$ are $3 \mathrm{ML}$ and $10 \mathrm{ML}$.

$=m_{0} /\left(\gamma_{1}-2 \gamma_{2}\right)$. The numerical calculations are based on the finite element technique on a variable size grid.

\section{NUMERICAL RESULTS AND DISCUSSIONS}

Before discussing the trion binding energies in a rectangular InAs/InP quantum wire, we present the results for the exciton binding energy. In Fig. 2, the height of the wire $h$ is varied between $3 \mathrm{ML}$ and $10 \mathrm{ML}$ and the width is fixed to $180 \AA$, the exciton binding energy increases because of the diminishing of the electron and the hole wave function radii when $h$ becomes smaller. However, for the very thin quantum wires, the opposite behavior for the exciton binding energy is found, namely, when $h$ decreases up to 3 ML the exciton binding energy decreases with decreasing $h$. The reason for this behavior is the electron wave function spillover effect $^{26,27}$ (compare the upper insets of Fig. 2, the contour plots of the electron wave functions for $h$ equal to $10 \mathrm{ML}$ and $3 \mathrm{ML})$. Note that for InAs/InP QWR with height $h=15 \AA$ and width $w=180 \AA$, we obtained a slightly higher binding energy $E_{X}^{B}=17.69 \mathrm{meV}$ than the one found in our earlier work ${ }^{27} E_{X}^{B}=13.79 \mathrm{meV}$, because in the present calculations we use the nonparabolic mass of the electron.

Further, we investigate the stability of the trions in selfassembled rectangular InAs/InP QWRs. The possibility of observing the negative (positive) trion depends on its stability against dissociation into an exciton and a free electron (hole). Therefore, we calculate the binding energies $E_{X^{-}}^{B}$ and $E_{X^{+}}^{B}$ for both negative and positive trions. The trion binding energy is defined as the energy of the trion $E_{X^{-}}$or $E_{X^{+}}$minus the energy of the exciton (including the energy of a free, unbound electron or hole). We found that the binding energy of the negative and the positive trion (see in the central inset of Fig. 2 the curves with the triangles and the squares, respectively) has the same dependence on the wire height as the binding energy of the exciton mentioned above. Besides, we conclude that the observed binding energy for $X^{+}$is nearly 1.3 times larger than the one for $X^{-}$, and they are in the range between 1.8 and $2.5 \mathrm{meV}$. At present, there are no experimental results on $X^{ \pm}$in this system to compare with.

In Figs. 3 and 4, we show the trion wave function of the ground and the first excited state for InAs/InP QWR with height $h=15 \AA$ and width $w=180 \AA$. As we can see from Fig. 3(b), the antisymmetric wave function of the first excited state of $X^{-}$is unbound; as on a large computational grid of $2000 \times 2000 \AA^{2}$, the wave function is nonzero only near the axis and vanishes only at the edges of the grid. When we increase the dimensions of the grid, the situation does not change. Nevertheless, the wave function of the first excited state of the positive trion is well confined inside the QWR with respect to the $z_{h 1}$ and $z_{h 2}$ axes [see Fig. 4(b)]. From this, we conclude that the $X^{-}$has only one bound state for the
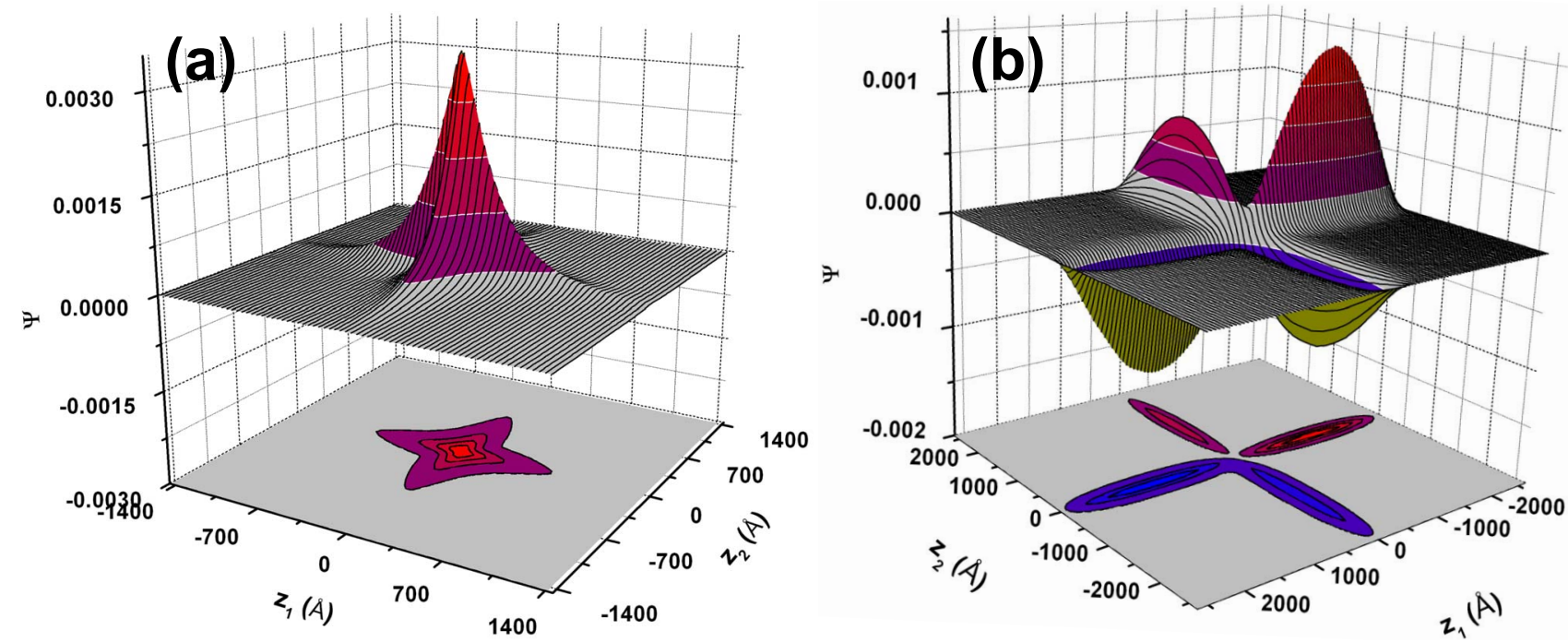

FIG. 3. (Color online) The wave function for relative motion of the ground (a) and the first excited (b) state for a negative trion $X^{-}$in a self-assembled InAs/InP QWR with height $h=15 \AA$ and width $w=180 \AA$. 

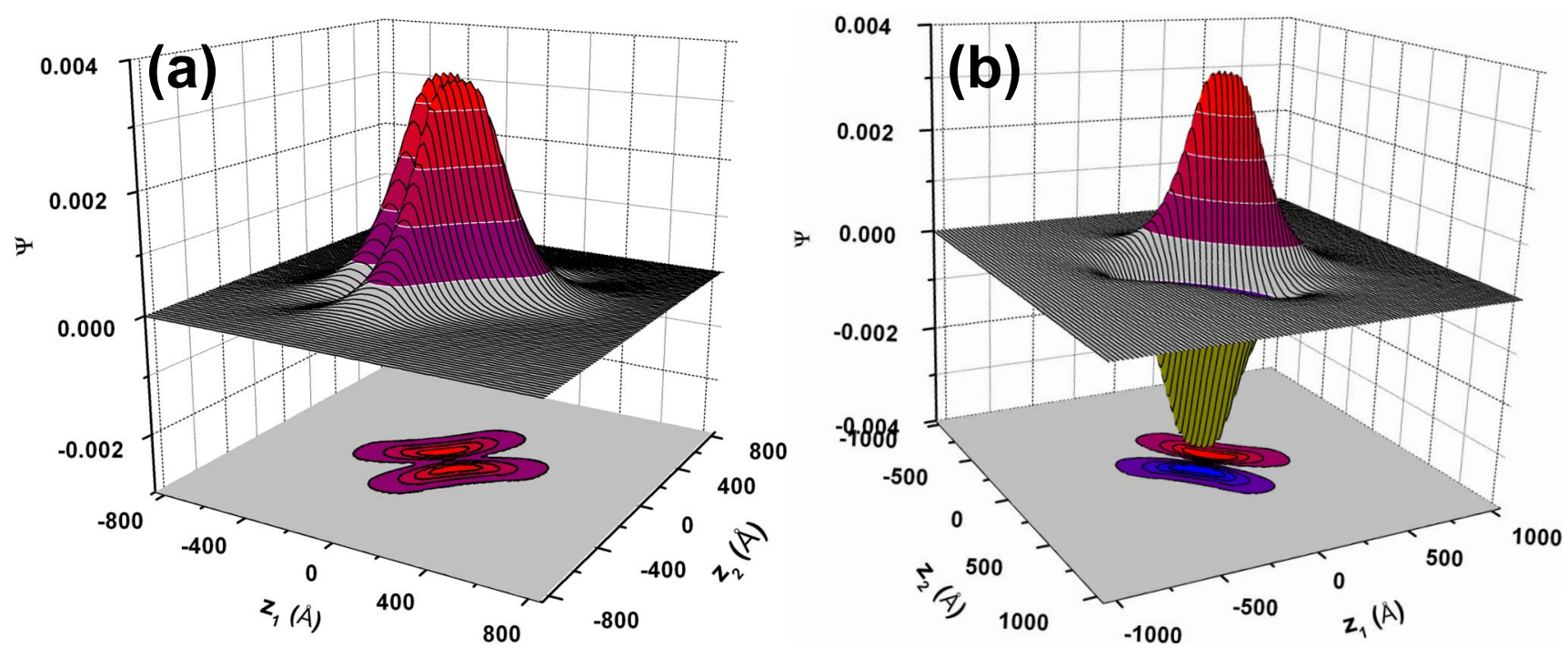

FIG. 4. (Color online) The same as Fig. 3 but now for the positive trion $X^{+}$.

chosen parameters of the QWR. Note from the contour plots that the ground state wave function has twofold symmetry and is symmetric with respect to the lines $z_{1}=z_{2}$ and $z_{1}=$ $-z_{2}$. The $X^{-}$ground state is more spread out than the $X^{+}$and the latter is almost zero along $z_{1}=z_{2}$ which implies that in the $X^{+}$ground state the probability to find both holes at the same spatial position is almost zero, while this is not the case for the two electrons in the case of the $X^{-}$. The wave function of the first excited $X^{ \pm}$state is symmetric with respect to $z_{1}=$ $-z_{2}$ and antisymmetric with respect to $z_{1}=z_{2}$ where the wave function exhibits a node. The $X^{+}$excited state is clearly bound with binding energy $2.61 \mathrm{meV}$.

Next, we examine the exciton and trion binding energy for a more complicated confinement geometry as realized in the V-shaped $\mathrm{GaAs} / \mathrm{Al}_{0.32} \mathrm{Ga}_{0.68} \mathrm{As}$ QWR. In Ref. 20, the thickness $h$ of the V-shaped QWR, measured at its center, was determined to be $70 \AA$ for the thick QWR sample. In order to investigate the dependence of the exciton energy on the height, we varied the GaAs/ $\mathrm{Al}_{0.32} \mathrm{Ga}_{0.68} \mathrm{As}$ QWR height $h$ in the range between 55 and $130 \AA$. In Fig. 5, we investigate the effect of the shape of the confinement potential by comparing the results for a V-shaped GaAs/ $\mathrm{Al}_{0.32} \mathrm{Ga}_{0.68} \mathrm{As}$ QWR [see the curve with the full circles (green) in Fig. 5] with those of a triangular shape of the GaAs/ $\mathrm{Al}_{0.32} \mathrm{Ga}_{0.68} \mathrm{As}$ QWR [see the curve with the open circles (red) in Fig. 5]. The exciton binding energy decreases as the height $h$ becomes larger in the case of a triangular shape of the wire. Such behavior of the exciton binding energy can be easily explained, since the electron (as well as the heavy hole which has a larger effective mass) is well confined inside the wire and its wave function becomes larger in size as the height of the wire $h$ increases, as shown in the top two graphs in the inset of Fig. 5. However, when we consider the V-shaped GaAs/ $\mathrm{Al}_{0.32} \mathrm{Ga}_{0.68} \mathrm{As} \mathrm{QWR}$, we found the opposite behavior for the exciton binding energy up to $h=100 \AA$, and beyond that point the binding energy slightly decreases when $h$ approaches the value of $130 \AA$. The reason is that for the wire with a smaller cross section (i.e., smaller $h$ and fixed width $w$ ), the electron (and the heavy hole) wave function is more strongly confined along the height direction, but less squeezed along the width direction (see the bottom two graphs in the inset of Fig. 5), which results in a decrease of the exciton binding energy.

Further, we discuss the formation of charged excitons in V-shaped GaAs $/ \mathrm{Al}_{0.32} \mathrm{Ga}_{0.68} \mathrm{As}$ QWR. In Fig. 6, we show the binding energy of the positive and negative trions as a function of the height $h$ of the GaAs/ $\mathrm{Al}_{0.32} \mathrm{Ga}_{0.68} \mathrm{As}$ QWR with width $w=510 \AA$. Similar to the binding energy for the exciton, we observe different behaviors for the binding energy for $X^{-}$and $X^{+}$depending on the shape of the wire. The binding energy of both negative and positive trions diminishes for the larger wires in case we consider a triangular-shaped wire. The binding energy of $X^{-}$and $X^{+}$increases for heights

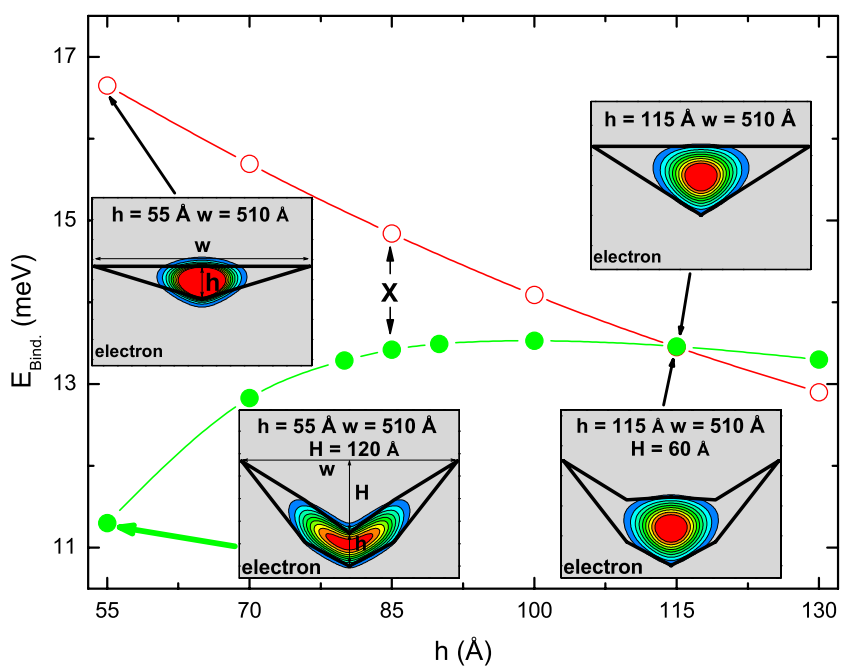

FIG. 5. (Color online) The exciton binding energy as a function of the height $h$ of the GaAs/GaAs/ $\mathrm{Al}_{0.32} \mathrm{Ga}_{0.68} \mathrm{As}$ QWR with $w$ $=510 \AA$. The curves with the open (red) and closed (green) circles correspond to the theoretical results when using a triangular and $\mathrm{V}$ shape of the wire, respectively, as shown in the insets. The insets show the contour plots of the electron wave functions for $h$ equal to 55 and $114 \AA$. 


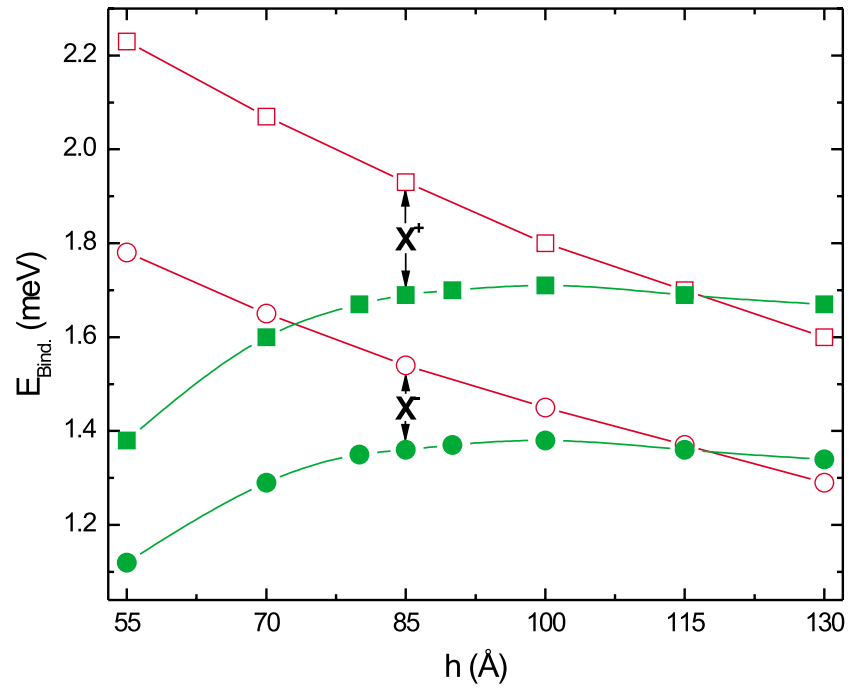

FIG. 6. (Color online) The same as Fig. 5 but now for the positive $X^{+}$and the negative $X^{-}$trion.

in the range between 50 and $100 \AA$ in case of the V-shaped GaAs $/ \mathrm{Al}_{0.32} \mathrm{Ga}_{0.68} \mathrm{As}$ QWR. Moreover, in Fig. 6, we can see that the binding energy for $X^{+}$is larger than the one for $X^{-}$, which means that the positive trion is more stable than the negative trion in $\mathrm{GaAs} / \mathrm{Al}_{0.32} \mathrm{Ga}_{0.68} \mathrm{As} \mathrm{QWR}$.

In Ref. 20, the binding energies of the positive trion $X^{+}$ and negative trion $X^{-}$were measured for the thin QWR. For the thick QWR, only the binding energy of the negative trion $X^{-}$was determined. The measured binding energies are listed in Table II. When comparing the calculated binding energies of $X^{+}$, we found that the thin wire results $E_{X^{+}}^{B}=2.85 \mathrm{meV}$ is in good agreement with the experimental binding energy. However, for $X^{-}$, we obtained in the case of the thin (thick) QWR a binding energy of $2.25 \mathrm{meV}(1.29 \mathrm{meV})$, respectively, which is about two times smaller than the experimental one $4.6 \mathrm{meV}$ (2.8) (see Table II). A possible reason for the enhanced $X^{-}$binding energy is that the $X^{-}$is probably localized along the unconfined wire direction as a consequence of
TABLE II. The numerical (Theor.) and experimental (Expt.) binding energy of the negative trion $E_{X^{-}}^{B}$ and the positive trion $E_{X^{+}}^{B}$ for thick and thin QWR samples.

\begin{tabular}{lcc}
\hline \hline & $E_{X^{-}}^{B}$ & $E_{X^{+}}^{B}$ \\
\hline Thin (Theor.) & 2.25 & 2.85 \\
Thin (Expt.) & 4.6 & 3.0 \\
Thick (Theor.) & 1.29 & 1.6 \\
Thick (Expt.) & 2.8 & \\
\hline
\end{tabular}

width and/or height fluctuations. Such fluctuations have been shown to enhance the $X^{-}$binding energy with about a factor of 2 in quantum wells. ${ }^{18,19}$ The effect of such fluctuations on $X^{-}$is much more pronounced than on $X^{+}$because electrons are lighter; therefore, they will be more strongly influenced by such local fluctuations than holes. However, this cannot explain the discrepancy in the trend that experimentally $X^{-}$ has a larger binding energy than $X^{+}$which is opposite to that found from our calculation. This is still an unsolved problem and will need further study.

\section{CONCLUSIONS}

We calculated the binding energy and wave function of trions in a QWR. Our numerical results predict the formation of negative and positive trions in self-assembled InAs/InP QWRs. We theoretically confirm the stability of both trions $X^{ \pm}$in $\mathrm{V}$-shaped $\mathrm{GaAs} / \mathrm{Al}_{0.32} \mathrm{Ga}_{0.68} \mathrm{As}$ wires and compared the binding energy to those experimentally measured. We found a strong dependence of the $X^{ \pm}$binding energy on the shape and the size of the QWR. The experimentally found larger binding energy for the $X^{-}$than for the $X^{+}$is not confirmed by our theoretical calculations and needs further investigation.

\section{ACKNOWLEDGMENTS}

This work was supported by the Belgian Science Policy (IAP), the Flemish Science Foundation (FWO-V1) and the European Commission network of excellence: SANDiE.

\footnotetext{
*bart.partoens@ua.ac.be

†francois.peeters@ua.ac.be

${ }^{1}$ M. A. Lampert, Phys. Rev. Lett. 1, 450 (1958).

${ }^{2}$ B. Stébé and A. Ainane, Superlattices Microstruct. 5, 545 (1989).

${ }^{3}$ K. Kheng, R. T. Cox, M. Y. d'Aubigné, F. Bassani, K. Saminadayar, and S. Tatarenko, Phys. Rev. Lett. 71, 1752 (1993).

${ }^{4}$ B. Stébé, A. Moradi, and F. Dujardin, Phys. Rev. B 61, 7231 (2000)

${ }^{5}$ S. Glasberg, G. Finkelstein, H. Shtrikman, and I. Bar-Joseph, Phys. Rev. B 59, R10425 (1999).

${ }^{6}$ A. Wojs and P. Hawrylak, Phys. Rev. B 51, 10880 (1995).

${ }^{7}$ D. M. Whittaker and A. J. Shields, Phys. Rev. B 56, 15185 (1997).

${ }^{8}$ J. R. Chapman, N. F. Johnson, and V. N. Nicopoulos, Phys. Rev. B 55, R10221 (1997).
}

${ }^{9}$ A. Esser, R. Zimmermann, and E. Runge, Phys. Status Solidi B 227, 317 (2001).

${ }^{10}$ T. Tsuchiya, Int. J. Mod. Phys. B 15, 3985 (2001).

${ }^{11}$ J. Bellessa, V. Voliotis, R. Grousson, X. L. Wang, M. Ogura, and H. Matsuhata, Appl. Phys. Lett. 71, 2481 (1997).

${ }^{12}$ A. Hartmann, Y. Ducommun, E. Kapon, U. Hohenester, and E. Molinari, Phys. Rev. Lett. 84, 5648 (2000).

${ }^{13}$ C. Riva, F. M. Peeters, and K. Varga, Phys. Rev. B 61, 13873 (2000); 63, 115302 (2001).

${ }^{14}$ G. Finkelstein, H. Shtrikman, and I. Bar-Joseph, Phys. Rev. B 53, R1709 (1996); 53, 12593 (1996).

${ }^{15}$ A. J. Shields, J. L. Osborne, D. M. Whittaker, M. Y. Simmons, M. Pepper, and D. A. Ritchie, Phys. Rev. B 55, 1318 (1997); A. J. Shields, F. M. Bolton, M. Y. Simmons, M. Pepper, and D. A. Ritchie, ibid. 55, R1970 (1997).

${ }^{16}$ J. G. Tischler, A. S. Bracker, D. Gammon, and D. Park, Phys. 
Rev. B 66, 081310(R) (2002).

${ }^{17}$ L. C. O. Dacal, R. Ferreira, G. Bastard, and J. A. Brum, Phys. Rev. B 65, 115325 (2002).

${ }^{18}$ A. V. Filinov, C. Riva, F. M. Peeters, Y. E. Lozovik, and M. Bonitz, Phys. Rev. B 70, 035323 (2004).

${ }^{19}$ A. S. Bracker, E. A. Stinaff, D. Gammon, M. E. Ware, J. G. Tischler, D. Park, D. Gershoni, A. V. Filinov, M. Bonitz, F. M. Peeters, and C. Riva, Phys. Rev. B 72, 035332 (2005).

${ }^{20}$ T. Otterburg, D. Y. Oberli, M.-A. Dupertuis, N. Moret, E. Pelucchi, B. Dwir, K. Leifer, and E. Kapon, Phys. Rev. B 71, 033301 (2005).

${ }^{21}$ B. Alén, J. Martínez-Pastor, A. García-Cristobal, L. González, and J. M. García, Appl. Phys. Lett. 78, 4025 (2001).

${ }^{22}$ B. Alén, and J. Martínez-Pastor, L. González, J. M. García, S. I. Molina, A. Ponce, and R. García, Phys. Rev. B 65, 241301(R) (2002).

${ }^{23}$ B. Szafran, T. Chwiej, F. M. Peeters, S. Bednarek, and J. Adamowski, Phys. Rev. B 71, 235305 (2005).

${ }^{24}$ A. F. Slachmuylders, B. Partoens, W. Magnus, and F. M. Peeters, Phys. Rev. B 76, 075405 (2007).

${ }^{25}$ L. González, J. M. García, R. García, F. Briones, J. MartínezPastor, and C. Ballesteros, Appl. Phys. Lett. 76, 1104 (2000).

${ }^{26}$ J. Maes, M. Hayne, Y. Sidor, B. Partoens, F. M. Peeters, Y.
González, L. González, D. Fuster, J. M. García, and V. V. Moshchalkov, Phys. Rev. B 70, 155311 (2004).

${ }^{27}$ Y. Sidor, B. Partoens, and F. M. Peeters, Phys. Rev. B 71, 165323 (2005).

${ }^{28}$ F. Vouilloz, D. Y. Oberli, M.-A. Dupertuis, A. Gustafsson, F. Reinhardt, and E. Kapon, Phys. Rev. Lett. 78, 1580 (1997).

${ }^{29}$ M. Grundmann, O. Stier, and D. Bimberg, Phys. Rev. B 52, 11969 (1995).

${ }^{30}$ J. Singh, Semiconductor Devices: Basic Principles (Wiley, New York, 2001).

${ }^{31}$ F. B. Pedersen and Y.-C. Chang, Phys. Rev. B 55, 4580 (1997).

${ }^{32}$ A. Krotkus and Z. Dobrovolskis, Electrical Conductivity of Narrow-Gap Semiconductors (Vilnus, Mokslas, 1988).

${ }^{33}$ M. Levinshtein, S. Rumyantsev, and M. Shur, Handbook Series on Semiconductor Parameters (World Scientific, London, 1996), Vol. 1.

${ }^{34}$ U. Ekenberg, Phys. Rev. B 40, 7714 (1989).

${ }^{35}$ I. Vurgaftman, J. R. Meyer, and L. R. Ram-Mohan, J. Appl. Phys. 89, 5815 (2001).

${ }^{36}$ Y. Sidor, B. Partoens, F. M. Peeters, N. Schildermans, M. Hayne, V. V. Moshchalkov, A. Rastelli, and O. G. Schmidt, Phys. Rev. B 73, 155334 (2006). 